\title{
Challenges and Opportunities of Public Health Research in Nepal
}

\author{
Shrestha S \\ Department of Epidemiology \\ School of Public Health, University of Alabama \\ Birmingham, Alabama, USA
}

Humans have evolved over thousands of years and have adapted to overcome daily life challenges and periodic pandemics. In particular, human well-being has made great strides in the $20^{\text {th }}$ century by preventing, detecting and responding to health issues. The overall successes in health, such as controlling smallpox, polio and cholera should be commended, but the ongoing fight against HIV/AIDS, threats of Ebola, malaria, tuberculosis, and other epidemics, including those like the occasional cholera outbreak in our own backyard will keep us honest about our previous successes. As countries advance in social and economic developments, health status and issues evolve as a result of changing lifestyles, demographics, climate change and epidemiological transitions. For example, while infectious diseases are still a main cause of death in developing countries like Nepal, the paradigm has slowly shifted towards four major chronic diseases - heart disease, cancer, diabetes and chronic respiratory disease, as a result of longevity due to the cautious global fight against selective infectious diseases.

One could argue that most of the improvements in health have been concentrated in developed countries and have not translated to resource-limited countries like Nepal. While the concept of "global health" is important and has several definitions and underlying debates, as Dr. Karmacharya indicated in the last issue of KUMJ, the health issues of the people in Nepal can be best addressed by the people in Nepal. ${ }^{1-3}$ I agree and think that for Nepal the right and timely approach is to solidify the foundation for her own public health, "Nepal public health" rather than still consider it as "global health" as how others perceive the latter from afar. In essence, "public health" has no boundaries; however, it is often defined and dictated by tradition, culture, ritual and the taboos that come with them which makes it difficult to not only generalize to have "a one size fits all" approach, but could be the mainstay of health inequalities both between and within countries. ${ }^{1,2}$ International organizations such as the United Nations (UN), World Health Organization (WHO) and other academic and non-profit agencies can provide parallel assistance to fight against health issues; however, a country like Nepal needs to build an internal infrastructure in public health.

Generally, there are three mainstays to public health: a) health care service - most of the health posts, clinics and hospitals are established to diagnose and investigate health problems and health hazards in the community; b) prevention and education - most NGOs/INGOs and international agencies serve this purpose by monitoring health status and mobilizing health programs; c) research - the basic infrastructure for this is lacking in Nepal and strengthening this area could be instrumental to Nepal's development of "public health". The first two areas are well known in Nepal; however, the research aspect is relatively new and some perceive it as irrelevant in a country like Nepal. It is disappointing that even amidst the countless national level reports published from the government or national and international agencies, there is still a dearth of high-quality and reliable data on a number of major public health issues of Nepal. In several cases, data is incomplete and even the best available ones are based on extrapolations from neighboring countries or regions - the relevance and accuracy of which might be debated.

In the field of public health, we are often faced with many ongoing and emerging challenges to act on any given issue and make wise and ethical decisions. The purpose of research is to scientifically translate evidence into practice by providing new insights and innovative solutions to effectively and efficiently keep people healthy and safe. Further, research can complement the other two areas of public health, discussed above, if not strengthen them. The importance of research in public health is evident from the statement by Dr. Margaret Chan, the Director-general of WHO.

"Research gives us the dynamic power to overcome these forces. We have the scientific method on our side. And we have developed some convincing evidence and arguments as a result. But as I stated, we need more of the right kind of research, and now more than ever. We need to see health included in all policies, and we need research to make the case." 4 
Public health research within Nepal is needed to a) define the problem, b) understand the underlying cause of the problem and c) potentially find a short term and long term solution to the problem so that policy-makers and practitioners can make informed decisions. However, there are several basic challenges that need to be addressed as we move forward with exploring opportunities in public health research in Nepal.

1. Ethics and Protection - Public health research should be conducted ethically ensuring the protection of individual privacy and avoiding any potential for participant exploitation. It is important that participants are fully informed of the research and benefits and thus can provide informed consent, which can be challenging in a setting like Nepal where a large portion of the population is illiterate. Nepal Health Research Council (NHRC) has established national ethical guidelines for health research in Nepal. ${ }^{5}$ Even then, while NHRC can review the scientific research protocols to ensure compliance with the guidelines, each research institute should also form an internal ethics board to enforce these ethical guidelines as research progresses.

2. Financial Resource - Research requires funding, which is often difficult to acquire even for a novel and innovative research concept. However, as Laurie Garrett argues in her article, "The Challenge of Global Health", often funding might not be the problem but rather the disproportionate allocation of funding to fight a single disease (vertical approach focused on one disease rather than horizontal approach with multiple diseases) might be the issue with public health globally. ${ }^{6}$ Funding might be based on the priority of the funder and the importance of the disease at that particular time. The use and implementation of the funds should be transparent and justified, which is often not the case. ${ }^{7}$ In addition, Nepal needs to encourage private sectors and civil societies to be involved in public health research through charity and philanthropic trusts.

3. Local Resources - There are hidden gems within our traditional medicine, herbal medicine and other cultural rituals that have been passed on over generations for cure or prevention of the disease. However, their key ingredients or objectives have not been scientifically compared or analyzed in most cases; thus, missing opportunities to locally develop modern medicine and preventative practices that can be efficient and cost-effective in various ways. ${ }^{8}$ Nevertheless, it is of utmost importance to make sure that these local treasures are not exported, but rather initially investigated locally.

4. Infrastructure - Research needs to be conducted systematically with an organized and formal structure. Besides training and funding, research infrastructure should connect to societal needs and include four major dimensions: human capacity (expertise, management), physical space (office, clinic, quality laboratory system, data collection and storage), organization (competencies, frameworks, interpersonal and organizational relationships) and society and government (policies, systems, and outreach). All of these can vary based on the scope of each research project; however, a basic infrastructure can provide opportunities to expand or incorporate other research projects.

5. 'Glocalization' - As discussed above, "global health" is defined in the context of health worldwide, but Nepal needs to start thinking of it as local "public health" ${ }^{1,2}$ While foreign aid and expertise are needed, Nepalese health workers, researchers, and the government need to take the initiative and lead to tackle the health issues. "Glocalization", a hybrid concept first adapted in Japanese business, describes the adaptation of global services to address the local needs that would be the most appropriate approach. ${ }^{9}$ Although, a caution is needed in generalizing the applications from other parts of the world to local Nepali public health issues.

6. Knowledge Translation - Data, results and findings at all levels need to be shared with a) the scientific community in the form of peer reviewed reports or at conferences and b) the local communities, with translation into layman's terms. Informing, exchanging, educating and empowering people about health issues at the local and national level is as important as acquiring information internationally. Donors also have a responsibility to coordinate meetings, monitor and evaluate programs or research and exchange information. In the new era of information technology, social media such as facebook, twitter, and text-message can also play vital roles in dissemination of information.

7. Opportunities to Grow - Research and scientific findings should be awarded based on merit. It will be more cost - effective to train individuals locally and provide incentives through private agencies, institutions or government to help develop new young local researchers and grow the research program. It cannot be emphasized more that health programs need to be led locally by the people of Nepal.

8. Academics - Public health research should be part of the standard curriculum at academic institutions, at least in medical and public health schools. Data collection is a big part of research; however, it needs to be collected systematically, ethically and with a public health purpose. Several institutes in Nepal have partnered with various foreign institutes to collaboratively establish public health curriculum and others offering Bachelor in Public Health and Masters in Public Health are very encouraging, although most are in the infant stage. It would be important to include practical approaches with a focus on research to these curriculums 
9. Sustainability - There needs to be coherence between NGOs/INGOs, local and state government with International organizations that implement public health programs. A country like Nepal depends on services from various NGOs and INGOs who tend to be closely connected with the local communities, but these are only sustained as long as they are funded. These organizations need to work with the local and central government with the hope that their programs are sustained to serve the local people. As we have witnessed with several projects including hospitals in Nepal, often health services are immediately shut down as soon as the foreign aid is discontinued. With research too, investment of all monetary, manpower and other resources should be justified for a long term purpose.

Some or most of these issues have been tackled at various levels with differing successes, but these need to be considered collectively in order to spearhead public health research in Nepal. Lessons from severe acute respiratory syndrome (SARS) and recent Ebola outbreak have taught us that a strict "Westphalian public health" model will not solve health issues in this era. ${ }^{10}$ I strongly believe and recommend that despite all the challenges, building in-house research capacity, adapting a "glocalization" model through collaborative national and international efforts will provide Nepal with opportunities to meet public health challenges faster, better, and more cost effectively.

\section{REFERENCES}

1. Bentley ME, Vilet GV. Global Health Is (Local) Public Health. N C Med J. Sep-Oct 2010; 71(5):448-51.

2. Fried LP, Bentley ME, Buekens P. Global health is public health. Lancet. 2010; 375(9714):535-537.

3. Karmacharya BM. Potential of Global Health Education in Low-Income Settings. KUMJ. 2013; 11(4): $266-7$.

4. Chan M. Health research needed now more than ever. Available form: http://www.who.int/dg/speeches/2008/20081117b/en/.

5. National Ethical Guidelines for Health Research in Nepal And Standard Operating Procedures (January 2011), Nepal Health Research Council (NHRC), Ramshah Path, Kathmandu, Nepal.

6. The Challenge of Global Health. Available form: http://www.foreignaffairs.com/articles/62268/laurie-garrett/the-challenge-of-global-health.

7. Giri A, Khatiwada P, Shrestha B, Chettri RK. Perceptions of government knowledge and control over contributions of aid organizations and INGOs to health in Nepal: a qualitative study. Global Health. 2013; 9:1.

8. Kunwar RP, Shrestha KP, Bussmann RW. Traditional herbal medicine in Far-west Nepal: a pharmacological appraisal. J Ethnobiol Ethnomed. 2010 ; 6:35.

9. Robertson, Roland. (1995) "Glocalization: Time-space and Homogeneity- heterogeneity", M. Featherstone et al (ed) Global Modernities, London; Sage :25-44.

10. Fidler, DP. SARS: Political Pathology of the First Post-Wesphalian Pathogen. Journal of Law, Med Eth. 31: 485-505. 